\title{
Case Report \\ Transformation of T-Cell Acute Lymphoblastic Lymphoma to Peripheral T-Cell Lymphoma: A Report of Two Cases
}

\author{
Michael Markow, ${ }^{1}$ Abu-Sayeef Mirza $\left(\mathbb{D},{ }^{2}\right.$ Lia Perez, ${ }^{3}$ Haipeng Shao, ${ }^{4}$ Pedro Horna, ${ }^{5}$ \\ Claudio Anasetti, ${ }^{4}$ Lubomir Sokol, ${ }^{4}$ and Mohammad O. Hussaini ${ }^{4}$ \\ ${ }^{1}$ Department of Pathology, Ohio State University, Columbus, OH, USA \\ ${ }^{2}$ Department of Internal Medicine, University of South Florida, Tampa, FL, USA \\ ${ }^{3}$ Bone Marrow Transplant Program, Moffitt Cancer Center, Tampa, FL, USA \\ ${ }^{4}$ Moffitt Cancer Center, Tampa, FL, USA \\ ${ }^{5}$ Mayo Clinic, Rochester, MN, USA \\ Correspondence should be addressed to Abu-Sayeef Mirza; mirzaa@mail.usf.edu
}

Received 5 November 2017; Accepted 31 December 2017; Published 28 February 2018

Academic Editor: Salah Aref

Copyright (c) 2018 Michael Markow et al. This is an open access article distributed under the Creative Commons Attribution License, which permits unrestricted use, distribution, and reproduction in any medium, provided the original work is properly cited.

\begin{abstract}
Nonhepatosplenic/noncutaneous $\gamma \delta$ peripheral T-cell lymphoma (NHNC $\gamma \delta$ PTCL) represents a miscellaneous group of unrelated $\mathrm{T}$-cell lymphomas of which only isolated cases have been reported. We describe two cases of transformation from T-lymphoblastic leukemia/lymphoma to NHNC $\gamma \delta$ PTCL. Transformation into more aggressive disease is a rare event in T-cell lineage-derived hematologic malignancies compared to B-cell neoplasms. Nevertheless, both of our cases involved relapse as PTCL manifested with skin involvement and an overt shift from blastic morphology to large granular leukemia-like mature T cells. Among other notable molecular characteristics, expression of immature markers such as TdT was lost in both cases. Based on cytogenetics, phenotype, and morphology, both patients represent a novel phenomenon of clonal transformation from T-ALL to PTCL which has rarely been reported in the literature. Such transformation may carry important diagnostic and biological implications.
\end{abstract}

\section{Introduction}

Peripheral T-cell lymphomas (PTCLs) comprise a heterogeneous group of indolent and aggressive T-cell lymphomas that confer a variable prognosis [1]. T-cell receptor gammadelta $(\mathrm{TCR} \gamma \delta)$ PTCLs are characterized based on their expression of $\gamma \delta$ glycoproteins within the TCR complex rather than more common alpha-beta $(\alpha \beta)$ glycoproteins identified in normal T-cells and in most T-cell neoplasms [2]. $\gamma \delta$ T-cells are subdivided into $\mathrm{V} \delta 1$ and $\mathrm{V} \delta 2$ subtypes and have demonstrated capacity for cytotoxicity, memory, and antigen presentation [3]. Mature $\gamma \delta$ PTCLs have no clear etiology to date and can be divided into three categories based on location: hepatosplenic (HSTL $\gamma \delta)$, cutaneous (PCTCL $\gamma \delta$ ), and nonhepatosplenic/noncutaneous (NHNC $\gamma \delta$ PTCL). HSTL $\gamma \delta$ and PCTCL $\gamma \delta$ both carry a similarly dismal prognosis [4].

NHNC $\gamma \delta$ PTCL represents a miscellaneous group of unrelated T-cell lymphomas of which only isolated cases have been reported; thus, characterization remains limited. Reported sites of involvement include lymph nodes and mucosal sites (nasopharynx and intestine), as well as the larynx, thyroid, lung, breast, and testis [5-8]. NHNC $\gamma \delta$ subtypes of PTCLs generally behave more aggressively compared to $\alpha \beta$ counterparts with an exception of $\gamma \delta$ variant of T-cell large granular lymphocyte (LGL) leukemia [9].

No cytogenetics findings unique to NHNC $\gamma \delta$ PTCL have been reported [10]. i7q, if detected, is not specific to $\gamma \delta$ PCTLs [11]. Gene sequencing of $\gamma \delta$ PCTLs has demonstrated conservation of LGR4, C3AR1, and SCARF2 among all $\gamma \delta$ PTCLs [12]. Notably, the NHNC $\gamma \delta$ PTCLs had CCL19, $M M P 9$, and UBD mutations, whereas HSTL $\gamma \delta$ PCTLs did not. These mutations were also strongly conserved in the $\alpha \beta$ PTCLs studied. Although there is evidence that some variants of $\gamma \delta$ PTCLs respond in vitro to aurora kinase inhibitors, among all PTCLs, those with TCR $\gamma \delta$ have the worst prognoses [13-15]. 


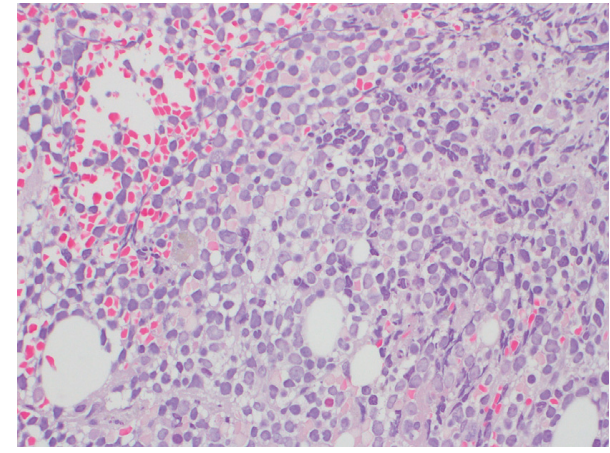

(a)

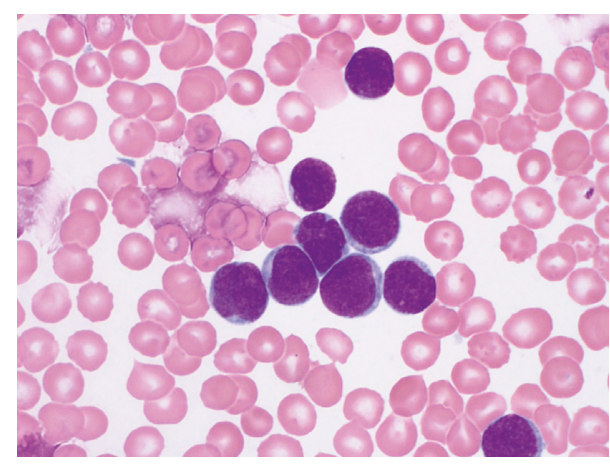

(b)

FIgure 1: Patient 1, bone marrow with T-cell ALL: (a) H\&E core biopsy, 500x; (b) aspirate cytology, 1000x.

As opposed to PTCL, the expression of $\gamma \delta$ TCR is relatively common in (up to 50\%) in T-lymphoblastic leukemia/lymphoma (T-ALL). Clinical features are similar to those that express $\alpha \beta$ TCR, and outcomes are comparable [8]. Here, we describe a case of transformation from T-ALL to NHNC $\gamma \delta$ PTCL. Despite being rarely reported, we also report a second case of PTCL arising in a patient with prior T-ALL. Such transformation may carry important diagnostic and biological implications.

\section{Case Presentations}

2.1. Patient 1. Patient 1 is a 31 -year-old gentleman diagnosed with T-cell ALL after presenting with diffuse petechial rash, cervical lymphadenopathy, abdominal pain, and a WBC of $121 \mathrm{~K} / \mu \mathrm{L}$ with $40 \%$ blasts (Figure 1). Cytogenetics showed 46, $\mathrm{XY}$,add(13) (p11.2)[3]/46,XY[17]. No BCR/ABL was detected by FISH; CSF was uninvolved. T-ALL was CD4+/CD8+, $\mathrm{CD} 2+, \mathrm{CD} 3+, \mathrm{CD} 5+(\operatorname{dim}), \mathrm{CD} 7+$, and $\mathrm{TdT}$ (dim). He was treated with vincristine, daunorubicin, pegylated L-asparaginase, and prednisolone achieving complete remission (CR). He was referred for hematopoietic stem cell transplant three months later which was delayed due to chemotherapy complications. During this period, he received leucovorin and glucarpidase. Prior to the transplant, a bone marrow biopsy was negative, but revealed clonal TCR $\beta$ and $\gamma$ gene rearrangements. In addition, CSF showed numerous LGLlike cells. The patient developed high grade fever and over one hundred erythematous plaques on his trunk, upper

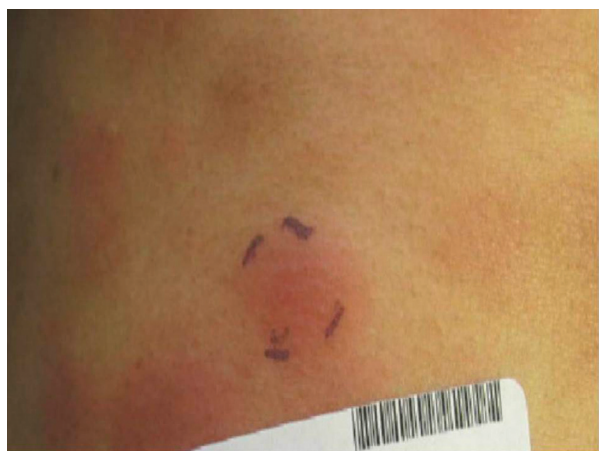

Figure 2: Patient 1, erythematous patches and plaques of PTCL distributed predominantly on torso and upper extremities.

extremities, and lower extremities (Figure 2). Skin biopsy showed NHNC $\gamma \delta$ PTCL (Figure 3) as did staging bone marrow biopsy (Figure 4). The malignant cells demonstrated mature chromatin, LGL-like morphology with prominent azurophilic granules resembling those seen in patient 2's PTCL (see below). They coexpressed CD4, CD8, CD56, cytotoxic markers, and $\gamma$ - TCR. TdT was negative. Molecular studies of the PTCL demonstrated that both the T-ALL and PTCL shared identical clonal TCR rearrangement peaks for both TCR $\beta$ and TCR $\gamma$ (Table 1).

Six months after initial CR, the patient was initiated on ICE therapy and intra-Ommaya reservoir methotrexate (later switched to intrathecal cytarabine). The skin lesions and CSF involvement initially resolved; however, he developed fevers, new generalized skin $\delta \gamma$-PTCL, nodal/hepatic progression by radiology, and CSF recurrence consistent with progressive disease. Bone marrow transplant was deferred, and the patient was admitted for hyper-CVAD chemotherapy. However, the patient requested transfer despite the worsening condition. Current clinical status is unknown.

2.2. Patient 2. Patient 2, deceased, was a 63-year-old Caucasian female with a past medical history significant for stage III invasive ductal carcinoma, ER/PR/HER2 negative, with 1 of 9 axillary lymph nodes positive for carcinoma, diagnosed at age 52. She underwent a left segmental mastectomy (lumpectomy) procedure with axillary lymph node dissection, radiation treatment, and 4 cycles of doxorubicin and cyclophosphamide chemotherapy. Other medical problems included hypertension, hyperlipidemia, herpes zoster, childhood rheumatic fever, menopause, and hypersensitivity pneumonitis.

She initially presented with a mediastinal mass, dyspnea, and pericardial and recurrent right-sided pleural effusions. Biopsy of the mediastinal mass revealed blasts with cyCD3+, $\mathrm{TdT}+, \mathrm{CD} 4+/ 8+, \mathrm{CD} 7+$ (Figure 5). TCR gamma gene rearrangement studies demonstrated clonal gene rearrangements. The peripheral blood and bone marrow were also involved.

She was treated with 18 cycles of hyper-CVAD followed by POMP maintenance for 18 months and remained in remission for nearly 4 years. Thereafter, she developed dyspnea, and radiographic imaging showed several simultaneous masses, including a $4.7 \times 4.0 \mathrm{~cm}$ right atrial mass and a left flank mass, in addition to pleural effusions. 


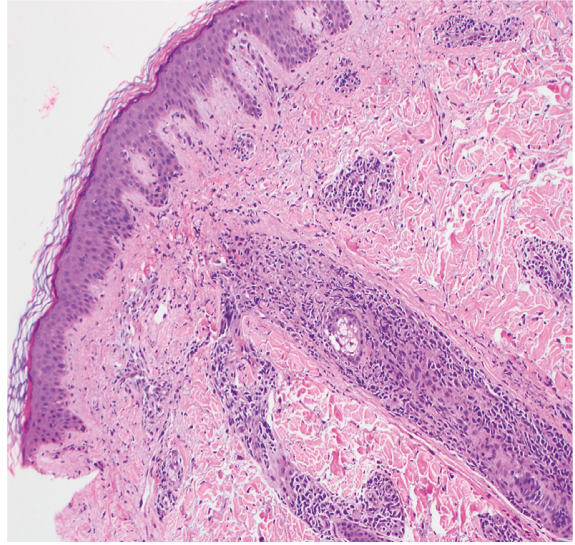

(a)

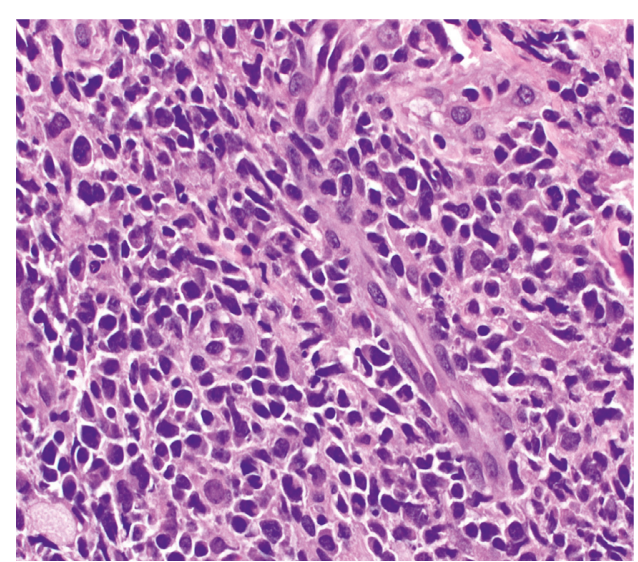

(b)

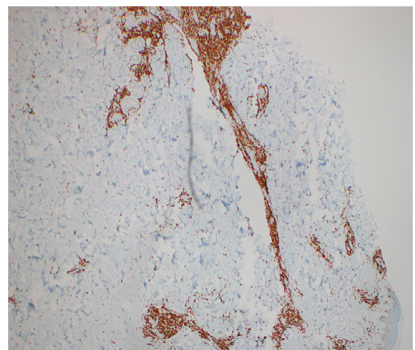

(c)

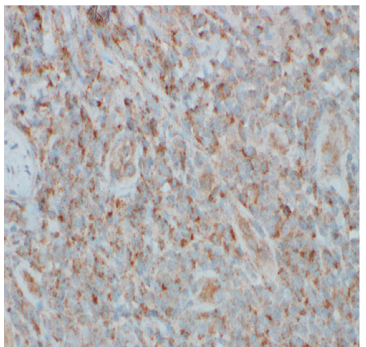

(d)

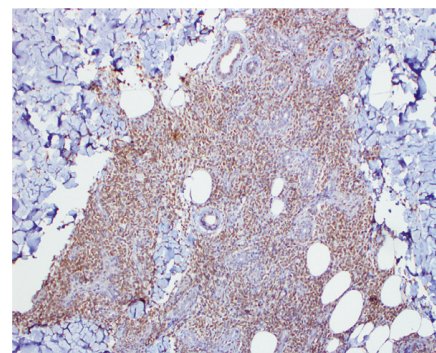

(e)

Figure 3: Patient 1, right flank skin with PTCL: (a) H\&E, 100x; (b) H\&E, 400x; (c) CD3, 100x; (d) TdT immunoperoxidase, 400x; (e) TCR gamma, 200x.

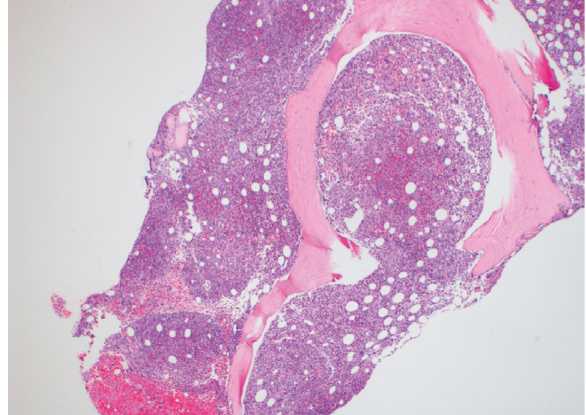

(a)

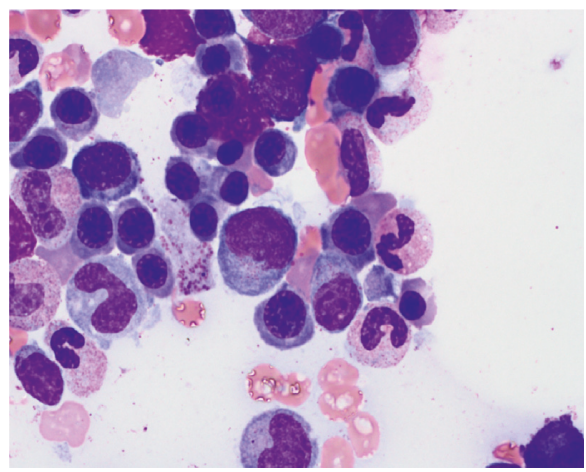

(c)

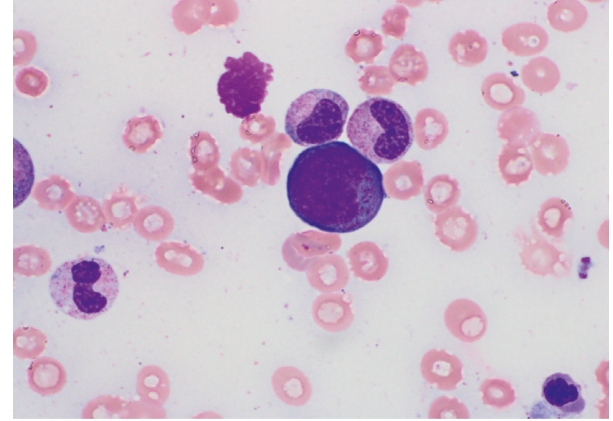

(b)

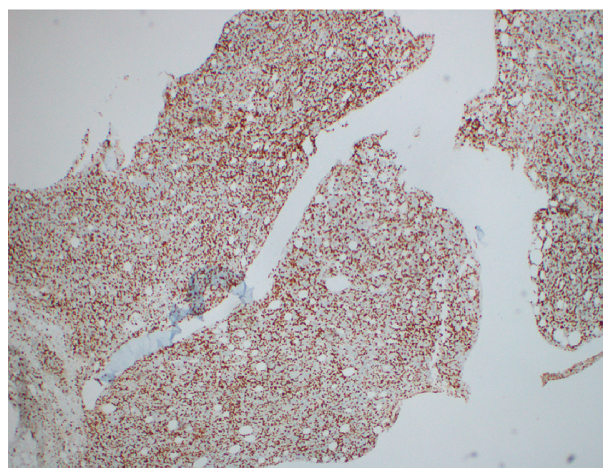

(d)

Figure 4: Patient 1, bone marrow with PTCL: (a) H\&E, 40x; (b, c) aspirate cytology, 1000x; (d) CD3, 100x. 


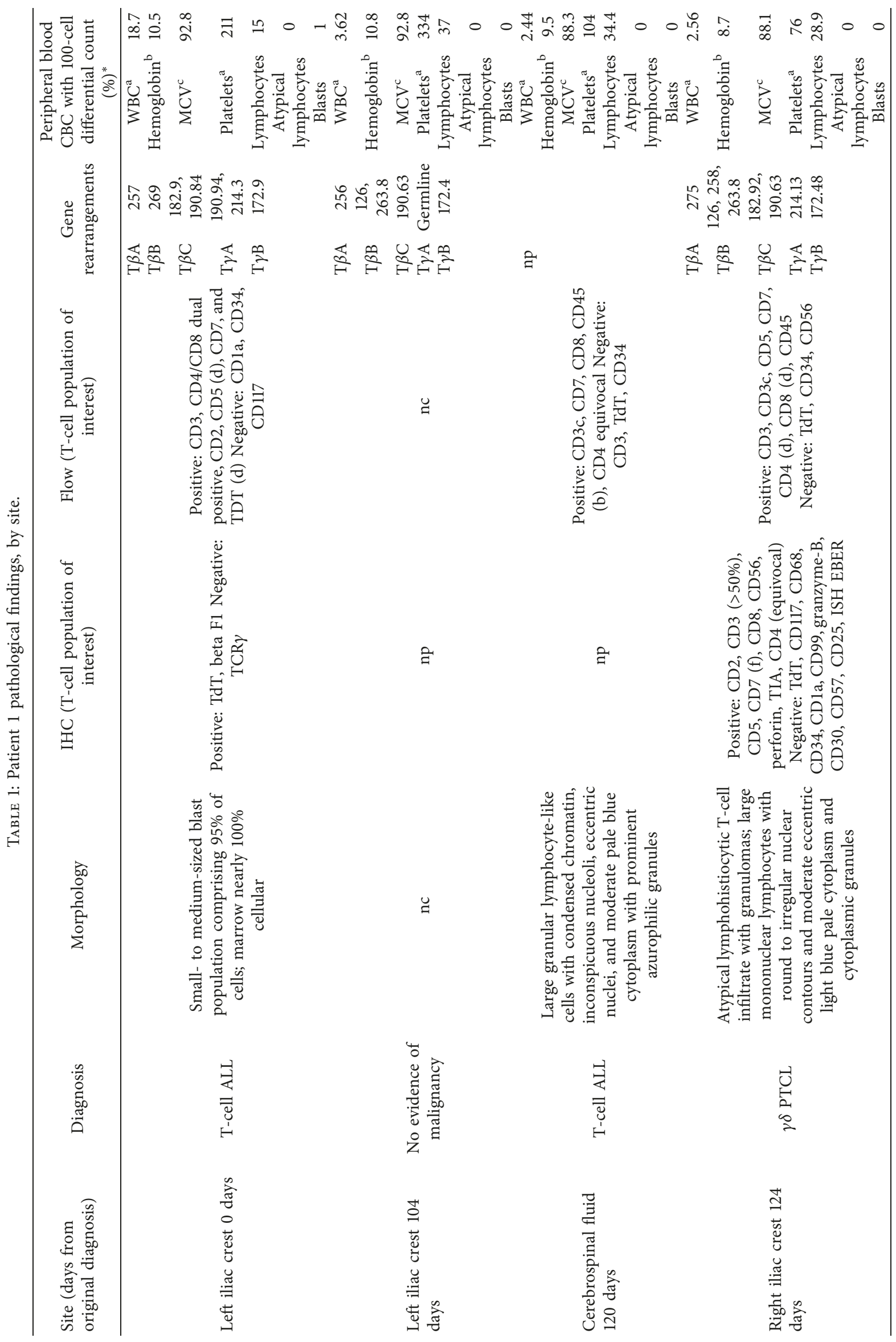




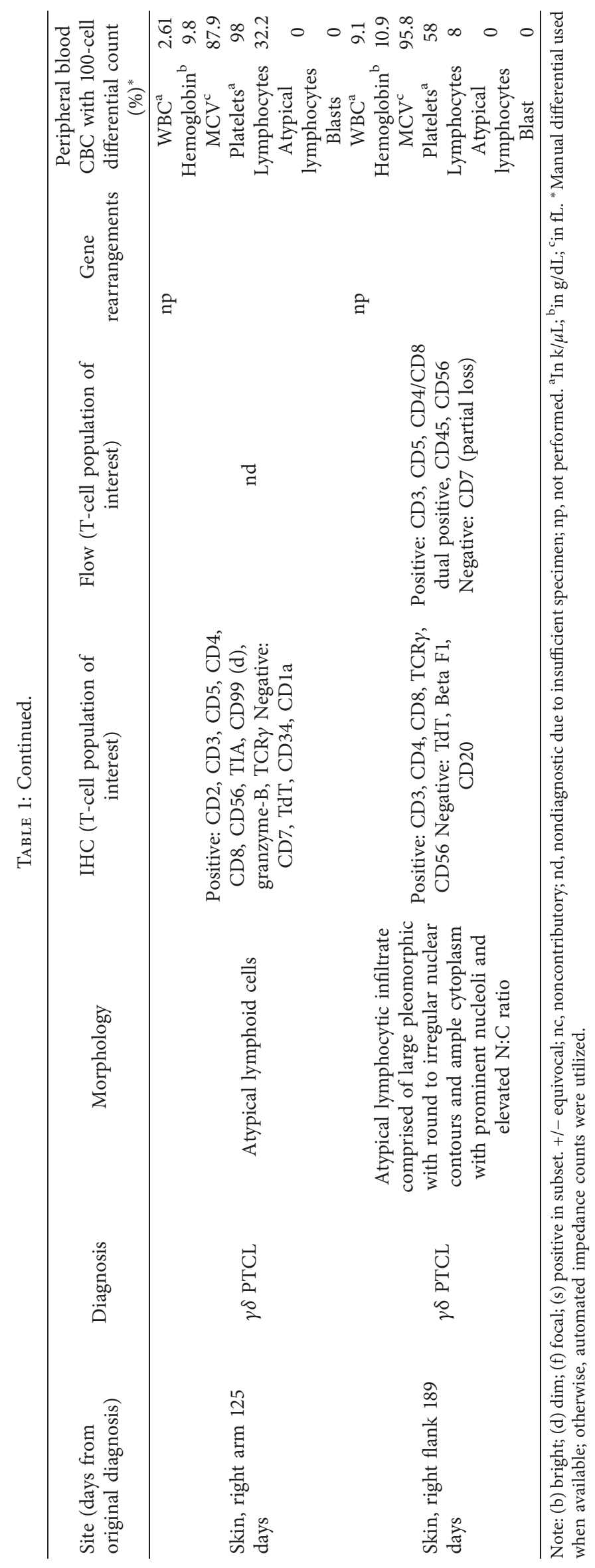




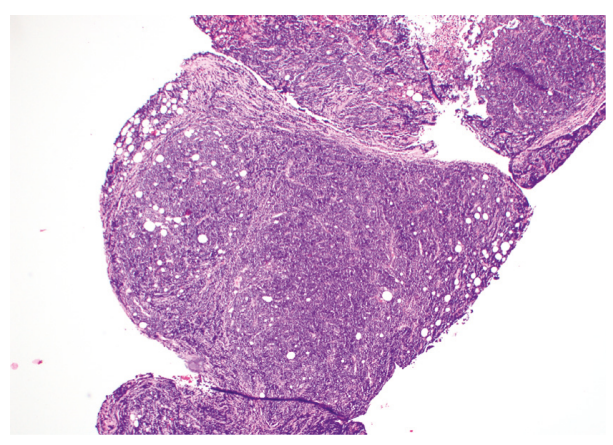

(a)

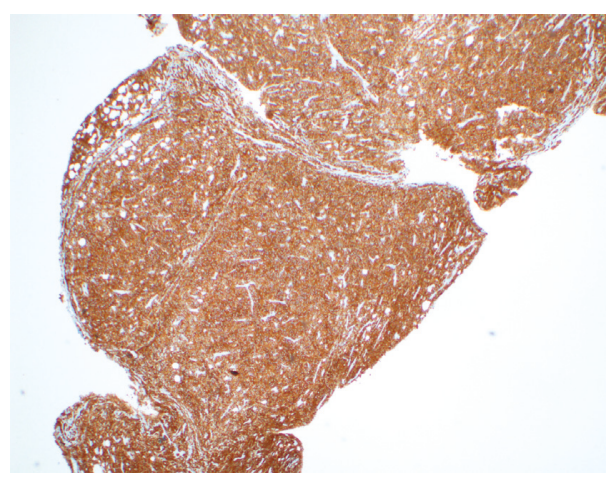

(c)

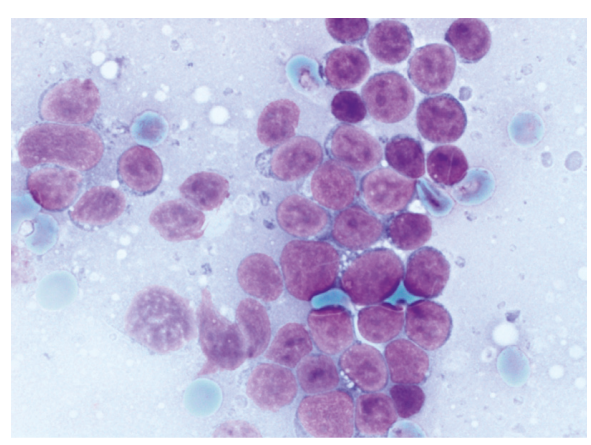

(b)

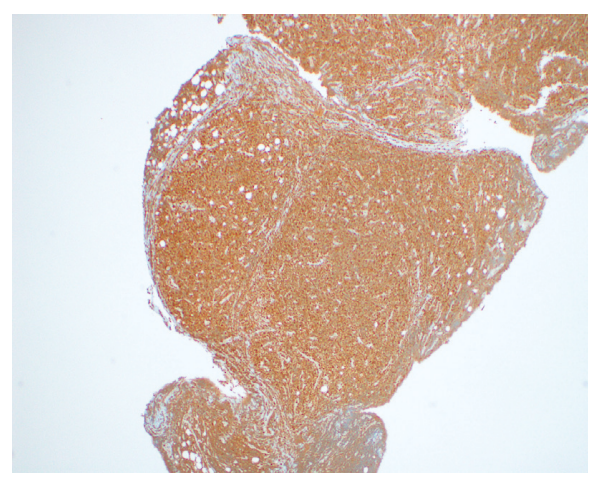

(d)

Figure 5: Patient 2; mediastinal mass with T-cell ALL: (a) H\&E, 100x; (b) cytology, 1000x; (c) CD3, 100x; (d) TdT, 100x.

Biopsy of the atrial mass was nondiagnostic showing myocardial and fibrous tissue. The chest wall (flank) mass was biopsied showing involvement by PTCL (Figure 6). Pleural fluid analysis showed involvement by $\mathrm{CD} 3+$ large lymphoid cells with markedly irregular nuclear contours, cytoplasmic azurophilic granules, lacking TdT and CD7, and showing positivity for CD8. Clonal TCR $\beta$ and $\gamma$ gene rearrangements were detected. Staging bone marrow biopsy showed no evidence of T-ALL.

However, clonal TCR $\beta$ and $\gamma$ gene rearrangements were detected with a possible similar peak to that detected in the pleural effusion sample (Table 2). Shared identical clonal TCR peaks were not noted between T-ALL and PTCL specimens, but comparison is limited by the fact that TCR testing was performed in different labs and may have used disparate primer sets and analysis parameters. The patient later received 2 cycles of ESHAP resulting in partial response. However, PET scan showed SUV of 11 in the cardiac area. Romidepsin salvage chemotherapy was administered. The patient continued to have large pleural effusions with respiratory issues and eventually opted for hospice. She died 4 months after her presentation with PTCL and 50 months after her original diagnosis of T-cell ALL, at age 64 .

\section{Discussion}

In both cases, there was relapse as PTCL manifested with skin involvement and an overt shift from blastic morphology to "LGL-like" mature $\mathrm{T}$ cells with ample cytoplasm and abundant azurophilic granules. Phenotypically, expression of immature markers such as TdT was lost in both cases. Both patients reasonably represent a novel phenomenon of clonal transformation from T-ALL to PTCL which has rarely been reported in the literature. In patient 1 , there is clear evidence of a clonal relationship as demonstrated by identical monoclonal TCR peaks. Also, both PTCL and T-ALL cases demonstrated the relatively immature CD4+/CD8+ immunophenotype. HSTL $\gamma \delta$ are often CD4-/CD8-, recapitulating normal $\gamma \delta \mathrm{T}$ cells, and less commonly C $8+/ C D 4$-is seen. In other $\gamma \delta$ PTCLs, CD4+/CD8phenotype may be observed perhaps deriving from a subset of normal $\gamma \delta \mathrm{T}$ cells that harbor this phenotype [8]. However, dual $\mathrm{CD} 4+/ \mathrm{CD} 8+$ has not been typically reported and is difficult to explain [16]. A clonal relationship is less clear for patient 2. However, the genesis of an entirely new PTCL in a patient with established T-ALL would be unusual indeed. TCR gene rearrangements cannot be reliably compared between the original T-ALL and PTCL due to their performance in differing laboratories.

Morphologically, $\gamma \delta$ PTCLs are a heterogeneous group. However, in both our cases we find a "LGL-like" morphology with large cells with ample cytoplasm containing cytoplasmic granules further supporting the notion that a common phenomenon may be at play in these two cases. Furthermore, SNP microarray performed on patient 2's PTCL showed clonal alterations in $95 \%$ of the cells, but clonal evolution was seen in $41-55 \%$ of the cells suggesting further that this represents a type of transformation. Furthermore, homozygous interstitial deletion 14q32.2 was detected which houses $B C L 11 B$. BCL11B encodes a transcription factor 


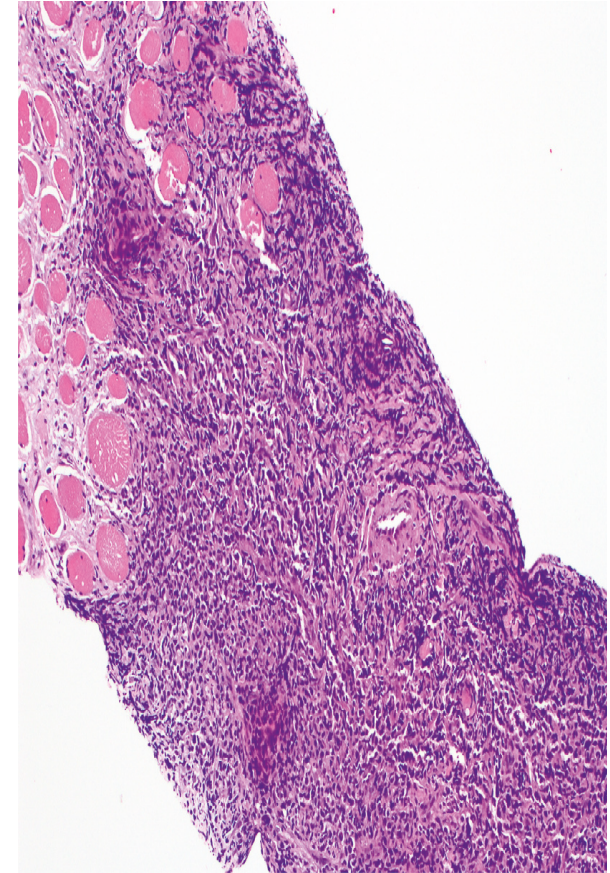

(a)

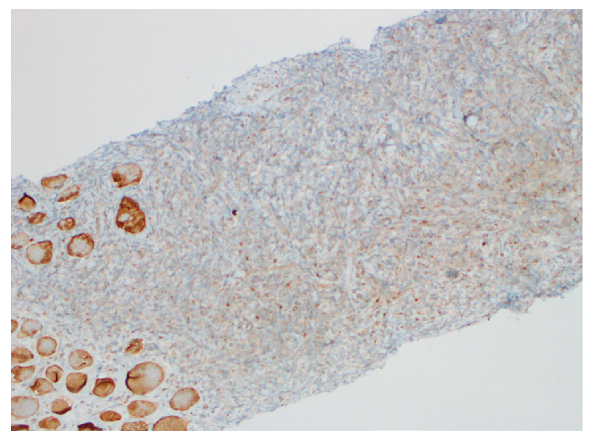

(b)

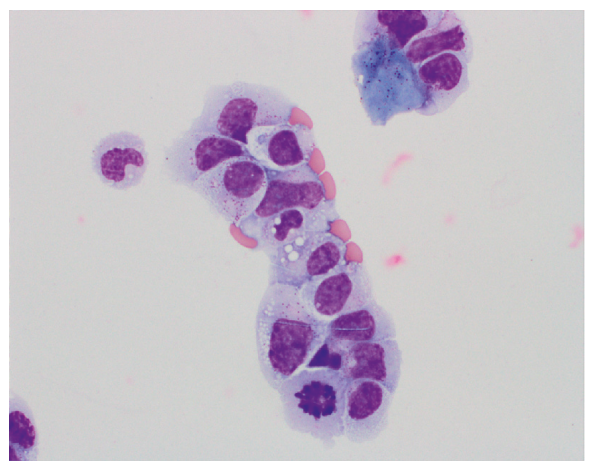

(c)

Figure 6: Patient 2: (a) flank mass with PTCL, H\&E, 100x; (b) flank mass, TdT immunoperoxidase, 200x; (c) pleural fluid cytospin cytology, 500x.

necessary for normal T-cell development and has been implicated in T-ALL pathogenesis [17]. Another line of evidence that supports the idea that these cases may represent a novel phenomenon is the detection of aberrations occurring at similar genetic loci in both cases. In patient 1 , karyotype showed 46,XY,add (13) (p11.2) [3]/46,XY[17] in the original T-ALL. In patient 2, SNP array performed on PTCL containing pleural fluid showed aberration at a similar locus: 13p11.1q24.21(34,474,059-115,670,586)x1-2.

If this truly represents transformation, we must consider the pathophysiology of such conversion. It may be postulated that chemotherapy may have forced the original T-ALL cells to undergo some degree of maturation. However, the disparate chemotherapy regimens in both cases, lack of similar conversions in other patients treated with these typical chemotherapy regimens, and temporal distance of relapse in patient 2 (3-4 years) do not support this speculation. More likely, acquisition of additional mutations in T-cell differentiation genes may have created a genetic context to allow for such transdifferentiation. This is supported by SNP array analysis performed on patient 2's PTCL showing clonal evolution. Another possibility is that malignant clones of T-ALL and PTCL developed separately from a common precursor sharing an initial transforming event. Subsequently, T-cell ALL and PTCL could evolve secondary to additional and separate transforming events.

The transformation into more aggressive disease is a rare event in T-cell lineage-derived hematologic malignancies compared to B-cell neoplasms. A patient with $\gamma \delta$-variant of LGL leukemia who developed aggressive ALL-like disorder after about 20 years of an indolent course has previously been reported [16]. Interestingly, cytology of leukemic cells during aggressive phase of disease did not differ significantly from LGLs assessed during indolent phase of disease. However, LGLs of the aggressive leukemia revealed very high Ki-67 proliferation index of $80 \%$, and SNP array showed multiple genetic abnormalities most probably implicated in the transformation. This report supports our hypothesis that transformation in T-cell lymphomas/leukemia is a very rare event and that multiple acquired somatic mutations secondary to therapeutic or environmental factors likely facilitated the transformed phenotype.

Besides the pathophysiology, the clinical and biological nature of these transformed PTCLs can be considered challenging. Should these cases be considered "ALL-like" disorders given their putative clonal derivation from T-ALL or do they represent a new entity with unique biology? The second case was considered to be a new entity with unique biology, hence the aggressive, chemotherapy refractory course that resulted.

In summary, we report two cases of NHNC PTCL arising in patients with established diagnoses T-ALL which, in at least one case, is clonally related. This raises the possibility of a novel pathologic phenomenon with associated diagnostic and biological implications.

\section{Conflicts of Interest}

The authors declare that they have no conflicts of interest.

\section{Acknowledgments}

The authors thank the supporting staff at the Moffitt Cancer Center including the departments of Malignant Hematology and Hematopathology. 


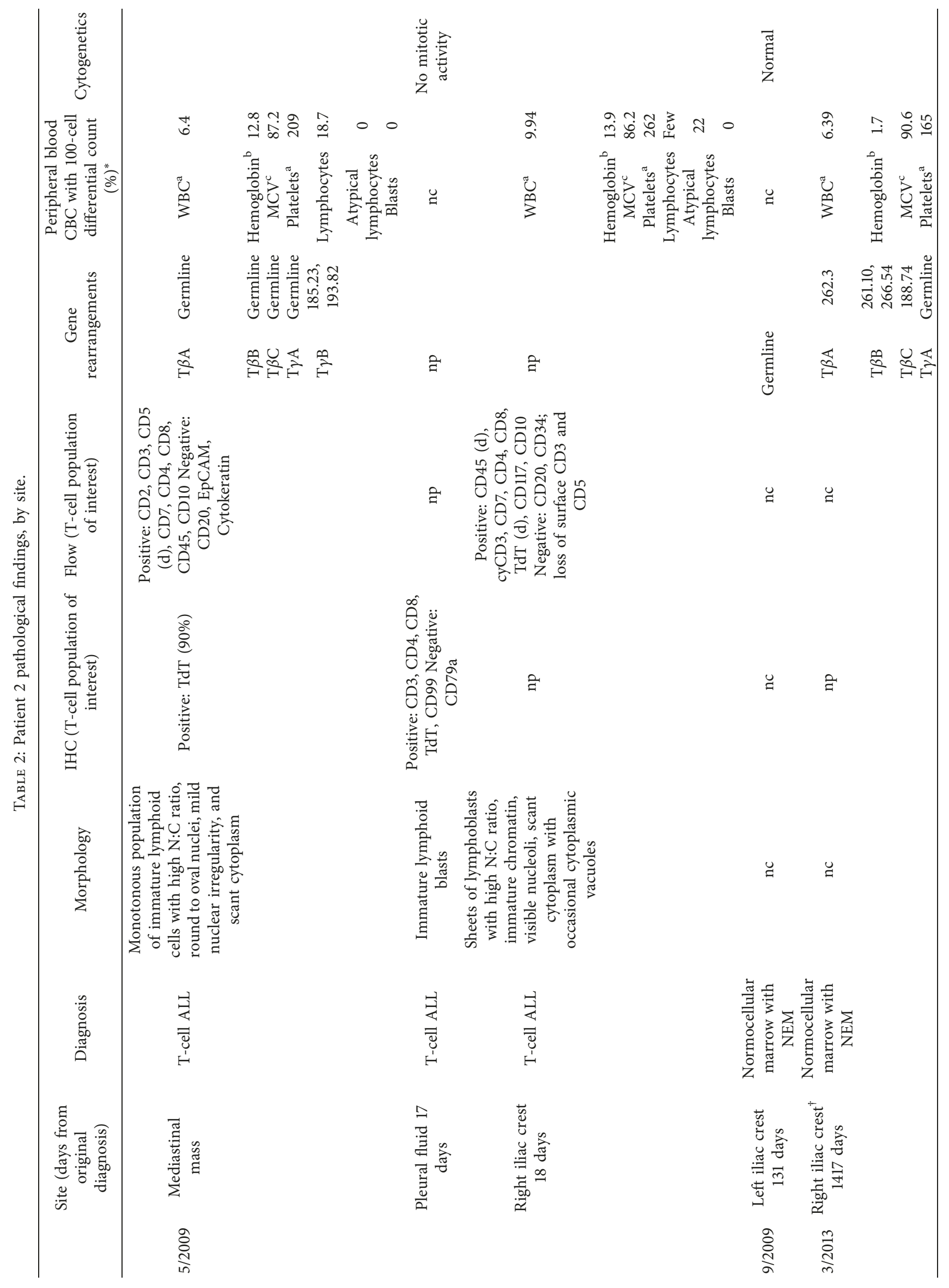




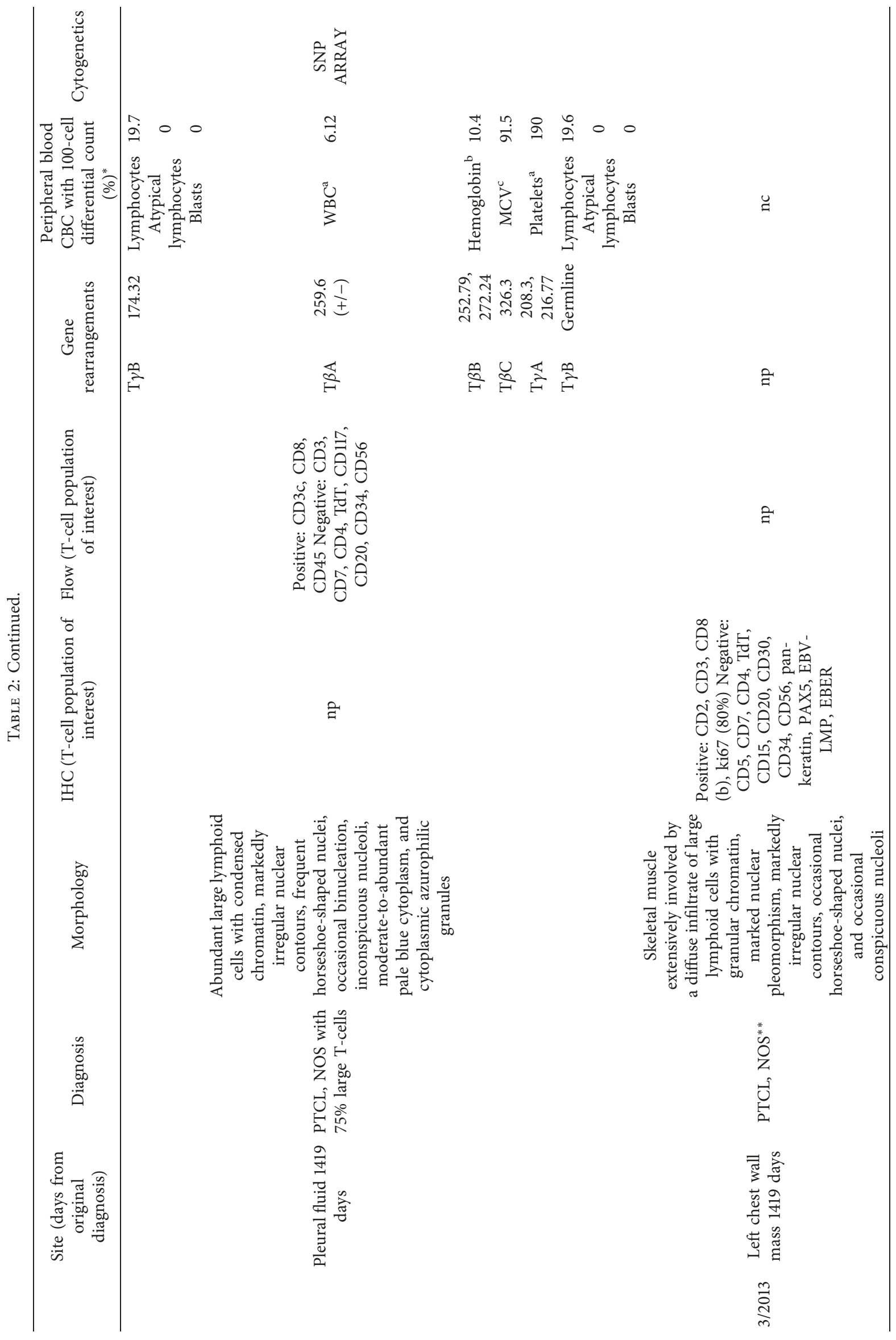




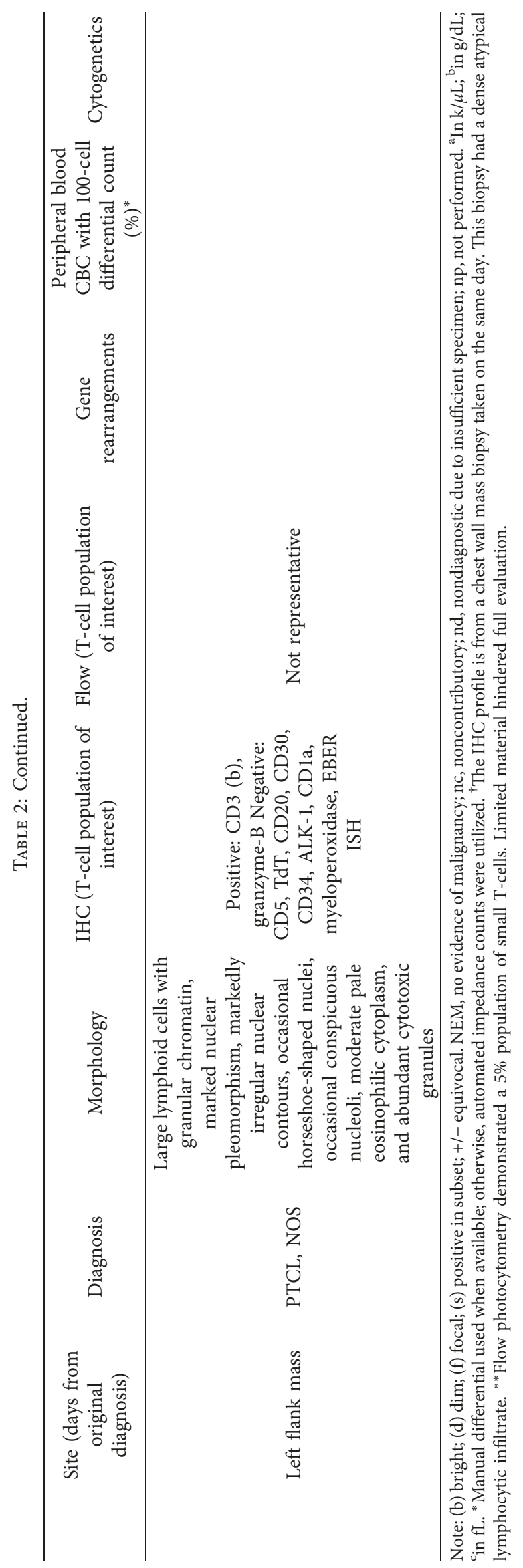




\section{References}

[1] E. A. Swerdlow, WHO Classification of Tumours of the Haematopoietic and Lymphoid Tissues, IARC, Lyon, France, 4th edition, 2008.

[2] C. Terhorst, J. de Vries, K. Georgopoulos et al., "The T cell receptor/T3 complex," Year in Immunology, vol. 2, pp. 245253, 1986.

[3] M. Calvaruso, A. Gulino, S. Buffa et al., "Challenges and new prospects in hepatosplenic $\gamma \delta$ T-cell lymphoma," Leukemia \& Lymphoma, vol. 55, no. 11, pp. 2457-2465, 2014.

[4] P. Gaulard and L. de Leval, "Pathology of peripheral T-cell lymphomas: where do we stand?," Seminars in Hematology, vol. 51, no. 1, pp. 5-16, 2014.

[5] P. Gaulard, T. Henni, J. P. Marolleau et al., "Lethal midline granuloma (polymorphic reticulosis) and lymphomatoid granulomatosis. Evidence for a monoclonal T-cell lymphoproliferative disorder," Cancer, vol. 62, no. 4, pp. 705-710, 1988.

[6] T. Saito, Y. Matsuno, R. Tanosaki, T. Watanabe, Y. Kobayashi, and K. Tobinai, " $\gamma \delta$ T-cell neoplasms: a clinicopathological study of 11 cases," Annals of Oncology, vol. 13, no. 11, pp. 1792-1798, 2002.

[7] S. Al Omran, W. A. Mourad, and M. A. Ali, "Gamma/delta peripheral T-cell lymphoma of the breast diagnosed by fineneedle aspiration biopsy," Diagnostic Cytopathology, vol. 26, no. 3, pp. 170-173, 2002.

[8] P. Gaulard, K. Belhadj, and F. Reyes, " $\gamma \delta$ T-cell lymphomas," Seminars in Hematology, vol. 40, no. 3, pp. 233-243, 2003.

[9] A. S. Bourgault-Rouxel, T. P. Loughran Jr., R. Zambello et al., "Clinical spectrum of $\gamma \delta+$ T cell LGL leukemia: analysis of 20 cases," Leukemia Research, vol. 32, no. 1, pp. 45-48, 2008.

[10] S. Lepretre, G. Buchonnet, A. Stamatoullas et al., "Chromosome abnormalities in peripheral T-cell lymphoma," Cancer Genetics and Cytogenetics, vol. 117, no. 1, pp. 71-79, 2000.

[11] F. Vega, L. J. Medeiros, and P. Gaulard, "Hepatosplenic and other $\gamma \Delta$ T-cell lymphomas," American Journal of Clinical Pathology, vol. 127, no. 6, pp. 869-880, 2007.

[12] K. Miyazaki, M. Yamaguchi, H. Imai et al., "Gene expression profiling of peripheral T-cell lymphoma including $\gamma \delta$ T-cell lymphoma," Blood, vol. 113, no. 5, pp. 1071-1074, 2009.

[13] B. T. Tan, K. Seo, R. A. Warnke, and D. A. Arber, "The frequency of immunoglobulin heavy chain gene and T-cell receptor $\gamma$-chain gene rearrangements and Epstein-Barr virus in $\mathrm{ALK}^{+}$and $\mathrm{ALK}^{-}$anaplastic large cell lymphoma and other peripheral T-cell lymphomas," Journal of Molecular Diagnostics, vol. 10, no. 6, pp. 502-512, 2008.

[14] C. Schutzinger, H. Esterbauer, G. Hron et al., "Prognostic value of $\mathrm{T}$-cell receptor gamma rearrangement in peripheral blood or bone marrow of patients with peripheral T-cell lymphomas," Leukemia \& Lymphoma, vol. 49, no. 2, pp. 237-246, 2008.

[15] J. Iqbal, D. D. Weisenburger, A. Chowdhury et al., "Natural killer cell lymphoma shares strikingly similar molecular features with a group of non-hepatosplenic $\gamma \delta$ T-cell lymphoma and is highly sensitive to a novel aurora kinase A inhibitor in vitro," Leukemia, vol. 25, no. 2, pp. 348-358, 2011.

[16] L. Zhang, R. Ramchandren, P. Papenhausen, T. P. Loughran, and L. Sokol, "Transformed aggressive $\gamma \delta$-variant T-cell large granular lymphocytic leukemia with acquired copy neutral loss of heterozygosity at 17q11.2q25.3 and additional aberrations," European Journal of Haematology, vol. 93, no. 3, pp. 260-264, 2014.

[17] A. Gutierrez, A. Kentsis, T. Sanda et al., "The BCL11B tumor suppressor is mutated across the major molecular subtypes of T-cell acute lymphoblastic leukemia," Blood, vol. 118, no. 15, pp. 4169-4173, 2011. 


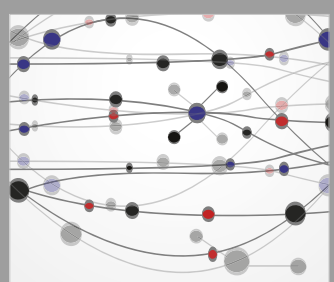

The Scientific World Journal
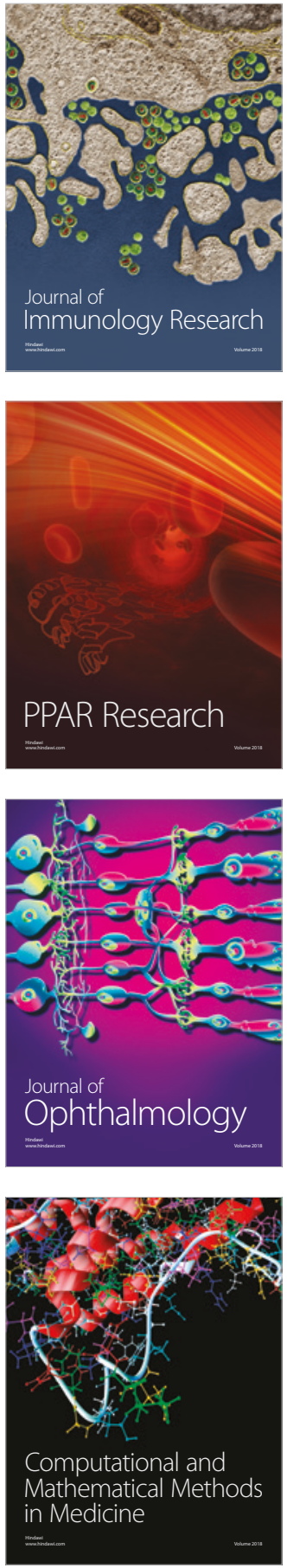

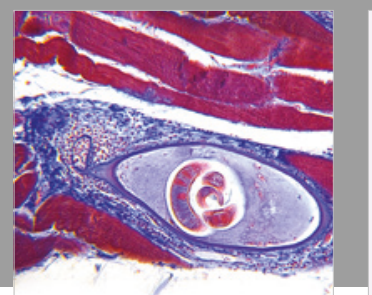

Gastroenterology Research and Practice

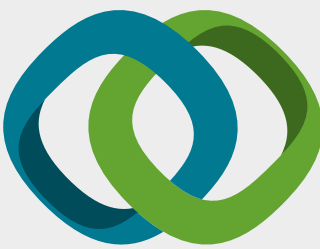

\section{Hindawi}

Submit your manuscripts at

www.hindawi.com
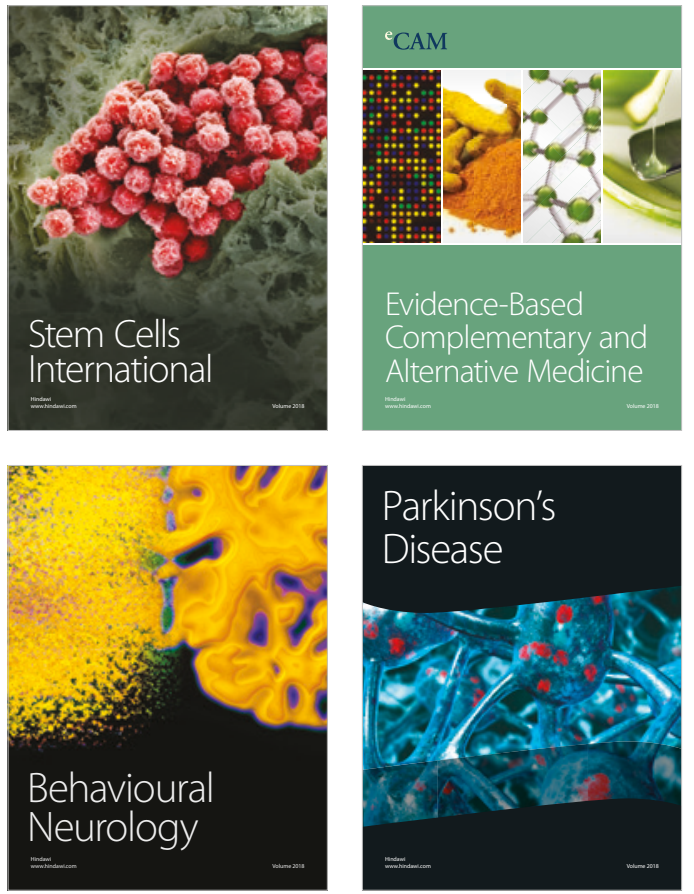

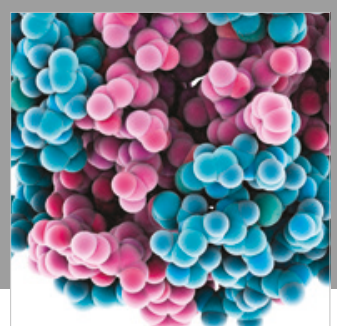

ournal of

Diabetes Research

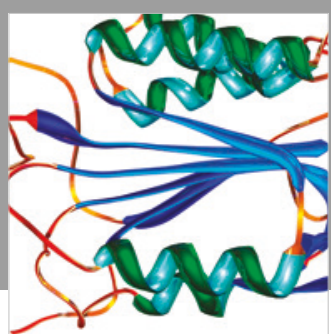

Disease Markers
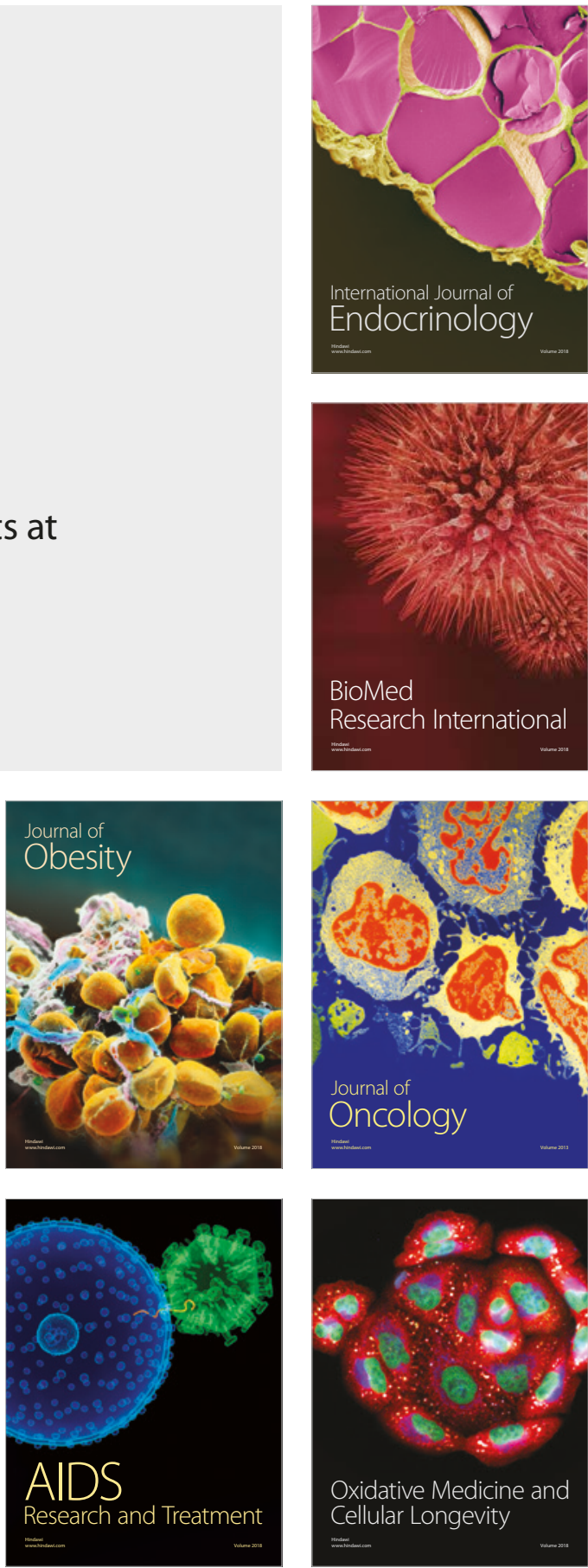